\title{
DE LA SÉPARATION AUX MOBILITÉS
}

\author{
Changer de regard sur l'occupation israélienne en Palestine
}

Stéphanie Latte Abdallah (Centre de Recherches Internationales, CNRS, Sciences Po, Paris)

Cédric Parizot (Institut de Recherche et d'Etudes sur le Monde Arabe et Musulman, UMR7310, CNRS/Aix Marseille Université, 13094 Aix en Provence, France.)

\begin{abstract}
Manuscrit auteur de l'introduction du livre Israël Palestine : L'illusion de la séparation, 2017, à paraître en septembre 2017. Marseille, Presses universitaire de Provence

La table des matières de l'ouvrage est présentée en fin $d^{\prime}$ introduction
\end{abstract}

La politique de séparation israélienne mise en œuvre depuis les années 1990 n’a pas coupé les relations entre Israéliens et Palestiniens, elle les a profondément transformées. Partant de ces interactions et des espaces qu'elles structurent, ce livre propose d'apporter une compréhension plus nuancée du fonctionnement du régime d'occupation et des frontières entre Israël, la Cisjordanie et la bande de Gaza. En outre, en proposant un détour par les expériences d'autres populations (Palestiniens du Sud Liban, Libanais de Galilée, migrants venus d'Afrique et d'Asie) ce livre souligne combien le discours sur la séparation masque d'autres constructions et pratiques des espaces israélo-palestiniens.

Cet ouvrage prend ainsi le contrepied des approches dominantes sur cette région. Plutôt que de se limiter à une étude institutionnelle des mécanismes de contrôle israéliens, ou de se focaliser sur leur impact au sein de la société palestinienne, il propose une perspective intermédiaire. Car l'analyse des vécus, des adaptations et des détournements que font les populations à partir de ces mécanismes de contrôle permet de mieux comprendre non seulement comment ce régime les affecte, mais également comment il est affecté par leurs réactions.

Ce livre rassemble quinze enquêtes de terrain menées par des historiens, des anthropologues, des géographes, des politistes et des sociologues. Ayant travaillé à la fois du côté palestinien, du côté israélien et dans les zones situées à l'interface de ces espaces, ces chercheurs mettent en lumière des processus qui permettent de mieux comprendre le fonctionnement du régime de mobilité israélien. Nous entendons par "régime de mobilité », l'ensemble des institutions, des réglementations, des infrastructures et des pratiques qui, depuis les années 1990, ont permis aux Israéliens de réguler, canaliser et surveiller les circulations quotidiennes des Palestiniens entre la bande de Gaza, Israël, Jérusalem Est et la Cisjordanie.

L'approche que nous mettons en œuvre dans ce livre s'inscrit donc dans la suite de celle que nous avions développée dans un précédent ouvrage À l'ombre du mur, Israéliens et Palestiniens entre 


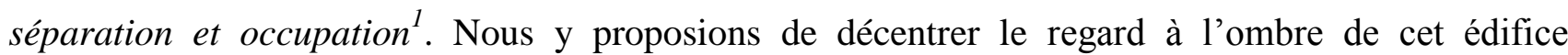
emblématique pour mieux envisager les dynamiques qu'il cachait. Nous expliquions que captivés par le Mur, les observateurs ne faisaient plus attention aux processus et aux reconfigurations du régime d'occupation israélien en Cisjordanie et dans la bande de Gaza (Latte Abdallah et Parizot, 2011, p. 15). Nous proposons ici d'aller plus loin en soulignant que c'est l'idée même de séparation territoriale qui engendre un regard biaisé sur ce conflit et ses espaces.

\section{L’illusion de la séparation territoriale}

L'idée de séparation territoriale s'inscrit dans un récit plus programmatique qu'explicatif. Et pour cause, elle est portée par le projet politique israélien de séparation promu par la gauche, puis par la droite depuis le début années 1990. Mise en scène à travers l'érection de murs, de checkpoints, ce projet a fortement mobilisé l'imaginaire de la frontière-ligne. Il a donc donné à beaucoup l'illusion d'avoir dissocié deux entités nationales et territoriales. L'idée de séparation territoriale nous paraît d'autant plus problématique qu'elle confine le débat sur le territoire israélien, la Cisjordanie et la bande de Gaza, alors même que les échanges entre les habitants de cette zone, ceux des pays voisins (Jordanie, Égypte, Liban, Syrie, etc.), et les populations migrantes affectent elles aussi les trajectoires du conflit. La politique de séparation n'aurait jamais pu être amorcée au début des années 1990 si Israël n'avait pas recruté massivement des travailleurs étrangers pour s'affranchir de sa dépendance à l'égard de la main-d'œuvre palestinienne (Kemp et Raijman, 2008). Nous verrons d'ailleurs que cette politique a détourné l'attention de mécanismes de contrôle qui ont accentué l'imbrication entre les espaces des deux populations au point de compromettre chaque jour un peu plus la création d'un État palestinien viable aux côtés d'Israël.

\section{D’une séparation à l'autre : les évolutions du projet politique israélien}

Les premières mesures de séparation sont mises en œuvre au cours de la première intifada (19871993). Elles englobent alors l'instauration de permis de circulation, de couvre-feux, de bouclages, et le déploiement de checkpoints le long des axes routiers. Utilisées au départ pour contrôler et réprimer le soulèvement palestinien, ces mesures se structurent progressivement autour d'un projet politique plus systématique (Abu Zahra, 2007 ; Handel, 2009 ; Hanieh, 2006 ; Hass 2001, 2002). Celui-ci est notamment promu par Itzhaq Rabin, d'abord ministre de la Défense sous le gouvernement Shamir, puis Premier ministre de 1992 à 1995 (Arieli et Sfard, 2008). Cette politique jette les bases d'un système qui va progressivement remplacer le dispositif de type colonial qu'avaient instauré les Israéliens depuis la conquête de la Cisjordanie et la bande de Gaza en 1967 (Gordon, 2007, 2008).

Le processus de négociation dit «d'Oslo » (1993-1999) a conforté et légitimé la mise en œuvre de cette politique. La dissociation de deux entités territoriales et administratives, israélienne et palestinienne est envisagée comme une évolution nécessaire devant jeter les bases des futures frontières entre les deux peuples. Au gré des retraits des zones autonomes et semi-autonomes palestiniennes en Cisjordanie et dans la bande de Gaza, Israël délègue à l'Autorité palestinienne nouvellement créée l'administration des populations locales (Latte Abdallah et Parizot, 2011). D'ailleurs, la construction d'une barrière autour de la bande de Gaza et de points de passages dédiés aux personnes et aux marchandises suscite à l'époque peu de réactions au niveau local et international car, suivant le tracé l'ancienne ligne d'armistice, elle semble venir délimiter la future frontière entre Israël et la bande côtière.

\footnotetext{
${ }^{1}$ Cet ouvrage a été publié en 2011 chez Actes Sud. Nous avons publié une seconde version augmentée et mise à jour en anglais chez Ashgate en 2015 sous le titre, Israelis and Palestinians in the Shadows of the Wall, Spaces of Separation and Occupation.
} 
Cependant, cette réorganisation territoriale ne conduit pas au dessin des frontières tant attendues par les Palestiniens et certains acteurs internationaux. Inachevés, les négociations et les retraits partiels de l'armée israélienne font émerger un patchwork d'enclaves palestiniennes entourées de zones restées sous juridiction israélienne (Carte 1 [voir fichier: Carte1-intro-chap5]). Cette configuration va devenir d'autant plus complexe que les décennies suivantes seront marquées par une véritable réorganisation des mécanismes d'occupation civils et militaires.

Dans le contexte de l'échec du processus d'Oslo, du déclenchement du second soulèvement palestinien (septembre 2000) et de la montée sans précédent de la violence entre les deux parties (Enderlin, 2002 ; Smith, 2007), la majorité de l'opinion publique israélienne et la plupart des membres de la classe dirigeante du pays intègrent l'idée qu'il n'y a plus de partenaires pour les négociations et qu'il n’y aura plus de solution négociée au conflit (Cypel, 2005).

Élu en 2001, Ariel Sharon a alors toute la marge pour engager son pays sur la voie de l'unilatéralisme. En 2002, après avoir ré-envahi les enclaves autonomes palestiniennes, il met en œuvre la construction de ce qui est nommé la «barrière de sécurité » (gader bitakhon) ou «barrière de séparation » (gader hafrada). Son objectif est double : il s'agit d'une part de reprendre le contrôle des enclaves palestiniennes pour mettre fin à la rébellion et, d'autre part, de montrer à l'opinion publique qu'il met véritablement en œuvre cette séparation.

La construction du Mur fait converger les agendas politiques de la gauche et de la droite et cristallise de multiples fonctions. Elle est considérée comme une solution sécuritaire - pour stopper les attentats suicides ; comme une solution démographique - permettant d'éviter un renversement de la balance démographique en faveur des Palestiniens et assurer la pérennité du projet sioniste ; comme une solution politique - imposant unilatéralement les frontières d'Israël tout en annexant le maximum de territoires et de colonies ; et enfin, comme une solution culturelle - car elle est aussi appréhendée comme l'érection d'un rempart entre le «monde libre » et «l'obscurantisme» (Rabinowitz, 2003 ; Parizot, 2009). Le Premier ministre israélien organise ensuite en 2005 le désengagement ou plutôt la «coupure » (hitnatkut) de la bande de Gaza : 8500 colons en sont évacués et les bases militaires qui s'y trouvent redéployées.

Enfin, son successeur Ehoud Olmert, élu en mars 2006, prône le retrait des colonies israéliennes situées à l'est de la «barrière de sécurité ». À travers ce plan, dit de «convergence » il désirait imposer unilatéralement les frontières de l'État d'Israël et établir une séparation définitive avec la population palestinienne de Cisjordanie. Cependant, ce projet n'a jamais vu le jour. Le conflit qui a éclaté avec le Hezbollah au Liban et le Hamas à Gaza à l'été 2006 a en effet contribué à remettre en cause l'efficacité des retraits unilatéraux qui avaient eu lieu précédemment : du Liban en 2000 et de Gaza en 2005. Du point de vue de la plupart des Israéliens, ils n'ont pas réussi à apporter plus de stabilité sur les frontières du pays (Parizot, 2012).

Néanmoins, le projet global de séparation d'avec les Palestiniens n'est pas abandonné, et c'est une séparation sans retrait de l'occupation qui est dès lors envisagée. Cette perspective s'est largement imposée dans l'imaginaire national israélien, non seulement comme une solution au conflit, mais également comme un fait acquis. Pour beaucoup, c'est cette politique qui aurait mis fin à la seconde Intifada et aurait permis de faire chuter le nombre d'attentats-suicide. La disparition des ouvriers Palestiniens du paysage israélien et la possibilité de pouvoir rouler en sécurité sur les routes qui relient les colonies de Cisjordanie à Israël ont achevé de conforter les Israéliens dans l'idée que la politique de séparation a véritablement porté ses fruits et déplacé le conflit de l'autre côté du Mur (Parizot, 2009). 
Les trois autres affrontements particulièrement sanglants qui ont de nouveau opposé l'armée israélienne et les Palestiniens dans la bande de Gaza en 2008-2009, en 2012 puis en 2014 n'ont pas davantage remis en question cette vision. Les termes utilisés pour les décrire sont d'ailleurs très évocateurs : en hébreu, il n'est pas rare que l'on utilise le terme de «guerre » (milkhemet), comme si Israël était allé mener un conflit sur le territoire d'un État voisin.

Pour beaucoup d'Israéliens, ce statu quo est même une situation plus viable que le retour aux négociations (Parizot, 2010). Poussée jusqu'au bout par la droite israélienne, cette vision des choses a d'ailleurs été clairement affirmée par la majorité des ministres du gouvernement formé par Benyamin Netanyahou en 2015 lorsqu'ils ont exprimé à plusieurs reprises leur refus de création d'un État palestinien (FMEP Staff, 2015).

\section{Le projet de séparation territoriale à l'épreuve du terrain}

Sur le terrain, il n'y a pas de véritable séparation territoriale, car parallèlement aux déploiements de dispositifs de séparation depuis 2000, l'armée s'est efforcée de reprendre le contrôle des enclaves palestiniennes dont elle s'était retirée pendant les négociations d'Oslo. Elles a ainsi renforcé sa présence des deux côtés du Mur et déployé des mécanismes de contrôle jusqu'au cœur des zones palestiniennes.

Au cours de la seconde Intifada (2000-2004), l'armée a d'abord envahi les villes et villages palestiniens afin de s'attaquer directement aux groupes armés et aux forces de sécurité palestiniennes. Puis, tout en s'en retirant progressivement, elle a déployé un ensemble d'infrastructures de contrôle (tours de guets, barrières, tranchés, blocs de pierre, checkpoints, etc.) pour maintenir un étau autour de ces enclaves. L'objectif était triple : surveiller les activités des Palestiniens en leur sein, réguler les mouvements de personnes et de marchandises entre chacune d'elles (Ben Ari et al., 2004) et, surtout, se garder la possibilité d'y intervenir à tout moment (Amidror, 2007). Au cours de la seconde Intifada et des années suivantes, l'armée et la police israéliennes ont d'ailleurs mené de nombreuses opérations de renseignement (Razoux, 2006), des arrestations et des assassinats ciblés (Cohen, 2009 ; Latte Abdallah, 2011, 2012, 2015).

Depuis la fin de la seconde Intifada (2004), ces dispositifs de contrôle sont devenus de plus en plus sophistiqués, l'objectif étant de les rendre plus efficaces mais aussi moins visibles. L'occupation s'inscrivant dans la durée, les autorités israéliennes se sont efforcées de réduire son coût politique et économique (Havkin, 2011, 2015 ; Latte Abdallah, 2011, 2012, 2015). Par exemple, l'architecture, la localisation, l'organisation interne des checkpoints, ainsi que la terminologie pour les désigner ont été repensées pour conférer un caractère moins violent aux opérations de contrôle de la population palestinienne.

Parallèlement, la colonisation de peuplement s'est poursuivie à un rythme particulièrement soutenu. De 2002 à 2010, le taux d'accroissement du nombre de colons israéliens à l'est et à l'ouest du Mur était à peu près identique (FMEP, 2011a). En 2010, sur les 314132 colons installés en Cisjordanie - hors Jérusalem-Est (FMEP, 2013), 23 \% (soit 73 350) résidaient à l'est du mur (FMEP, 2011b). Depuis son élection en 2009, Benyamin Netanyahou a encouragé la poursuite de ce processus afin de miner davantage les conditions de création d'un État palestinien. Selon un rapport de Peace Now (2013), le Premier ministre israélien a approuvé la construction et les projets de nombreuses colonies à l'est du mur et à Jérusalem Est dont l'expansion rendra d'autant plus difficile 
la négociation d'un accord de paix. En 2012-2013, le nombre total de colons en Cisjordanie et à Jérusalem-Est était estimé à $547000^{2}$.

À ces systèmes d'occupation civils et militaires, il faut ajouter un ensemble de mécanismes moins visibles qui permettent à Israël de réguler l'acheminement des marchandises, des ressources et des informations vers et entre les enclaves palestiniennes. En maintenant le contrôle sur les principaux axes routiers, sur les checkpoints, mais aussi les ports et les aéroports par où transitent les marchandises palestiniennes, Israël conserve un levier qui lui permet de réguler les relations entre son économie et celles de la Cisjordanie et la bande de Gaza. Avant la période d'Oslo, Israël avait ainsi favorisé au sein de ces deux régions un processus de «dé-dévelopement ( Roy, 1999), pour ensuite les maintenir dans une situation de «marchés captifs » (Grinberg, 2011), voire de marchés pour le recyclage de produits périmés (Natsheh et Parizot, 2011). Le contrôle des systèmes d'alimentation en eau des zones palestiniennes (Trottier, 2007 ; Salenson dans ce volume), mais également en électricité ou en gaz ont également offert d'autres leviers décisifs aux Israéliens. Ils ont d'ailleurs été largement utilisés dans la mise en place du blocus autour de la bande de Gaza depuis 2007, pour faire pression sur la population gaziote et la mettre dans une situation de quasi crise humanitaire (Azoulay et Ophir, 2008). Enfin, en conservant le contrôle des infrastructures des télécommunications et celui de l'espace électromagnétique palestinien, les Israéliens conservent un pouvoir de régulation sur la circulation des informations entre les enclaves palestiniennes et entre celles-ci et l'extérieur (Dawes, 2015).

En bref, plutôt que d'instaurer une séparation territoriale entre les deux populations, les mécanismes de contrôle israéliens ont, depuis les années 1990, favorisé la mise en place un système « d'inclusion-exclusive » des Palestiniens (Ophir, Givoni et Hanafi, 2009). Fondé sur une régulation différentielle des mobilités des personnes et des marchandises, ce régime cherche à assurer la sécurité des Israéliens ainsi que la fluidité de leurs mouvements, en mettant à l'écart et en confinant les Palestiniens dans des espaces fragmentés. Alors que les colonies israéliennes et Israël sont restés un «archipel »d'îlots parfaitement connectés, les « enclaves » palestiniennes sont devenues de plus en plus isolées les unes des autres jusqu'à former un espace profondément discontinu (Petti, 2008). C'est un système de gestion des risques (Shamir, 2005) et de réduction de la friction qui cherche à compenser la proximité et l'imbrication des espaces de vie des populations israélienne et palestinienne.

Cette organisation différentielle de l'usage de l'espace et du temps a généré des rapports asymétriques entre les deux populations (Collins, 2008 ; Handel, 2009 ; Peteet, 2008 ; Petti, 2008). Elle a des implications politiques majeures puisqu'elle favorise l'émergence de perceptions de plus en plus décalées du conflit depuis la fin de la seconde Intifada (Parizot, 2009c, 2010). Évoluant dans un espace continu et pacifié, les Israéliens et la plupart des étrangers sont persuadés d'avoir été séparés des Palestiniens et que le conflit a été déplacé «de l'autre côté du Mur»; à l'inverse, confinés et contrôlés continuellement, les Palestiniens vivent un renforcement progressif de l'occupation et de la violence israélienne.

\section{Apartheid, colonialisme ou régime de mobilité contemporain?}

Dans nos deux précédents ouvrages (Latte Abdallah et Parizot, 2011, 2015), nous avions insisté sur le caractère très contemporain du système de contrôle israélien ainsi que sur les erreurs de lecture auxquelles pouvait conduire une assimilation sans nuances avec l'apartheid sud-africain. Nous expliquions que cette comparaison risquait non seulement d'engager la réflexion sur une voie plus

\footnotetext{
${ }^{2}$ Dont 350000 en Cisjordanie selon les chiffres données par B’Tselem, http://www.btselem.org [consulté le 16 juillet 2016].
} 
politique qu'analytique, et qu'elle tendait en outre à réduire ce régime à un système colonial anachronique et dépassé. Nous aimerions revenir ici sur cette question pour mieux rendre compte de la dimension heuristique de ces approches tout en soulignant leurs limites.

\section{Apartheid et colonialisme de peuplement}

Les analogies avec l'apartheid ne sont pas récentes. Les restrictions de mouvement imposées à la main-d'œuvre palestinienne et la création des enclaves autonomes administrées par l'Autorité palestinienne ont souvent été assimilées au système des bantoustans sud-africains (Farsakh, 2002, 2006 ; Legrain, 1996, 1997 ; Abu Zahra, 2007 ; Hanieh, 2006). Certains ont également souligné les similitudes structurelles au niveau des permis de circulation (proches des Pass Laws sud-africains) et des statuts distincts assignés aux différents groupes de populations (Dayan, 2009). En distinguant différents niveaux de citoyens, pseudo-citoyens et non-citoyens (Veracini, 2006, 2010), et différents degrés d'accès à la mobilité, ces systèmes ont organisé un régime frontalier domestique (Dayan, 2009).

Ces comparaisons ont été particulièrement stimulées par la construction du Mur dans les années 2000 et, surtout, par le durcissement de ce régime de séparation au cours de la seconde Intifada (Bishara, 2002 ; Veracini, 2006 ; Peteet, 2009 ; Yiftachel, 2009 ; Olmsted, 2009 ; Lentin, 2008 ; Dayan, 2009 ; Lebrun et Salingue, 2013 ; Pappé, 2015). Des représentants d'instances internationales (Dugard, 2007), des journalistes (Bôle-Richard, 2013), ainsi que des hommes politiques ont largement contribué à alimenter cette comparaison.

L'enjeu n'a pas simplement été académique mais il a aussi été politique puisqu'il s'agissait d'ouvrir un débat et de dénoncer un régime (Toensing, 2009). Il est enfin juridique dans la mesure où il permet de mettre Israël en cause pénalement. L'apartheid est en effet défini comme un crime par la Convention internationale sur l'élimination et la répression des crimes d'apartheid (1973) et reconnu par le statut de Rome qui a institué la Cour pénale internationale (2002).

L'assimilation du régime de mobilité israélien à un régime d'apartheid a aujourd'hui d'autant plus de résonnance que l'État palestinien a adhéré au Statut de Rome et à la Cour pénale internationale en avril 2015 et que des démarches visant à faire reconnaître un crime de cette nature peuvent être entreprises. Depuis quelques années, face à la droitisation constante des gouvernements israéliens, la mise en échec répétée des tentatives de négociations et leur soutien affiché au processus de colonisation, l'Autorité et la société civile palestiniennes ont choisi d'internationaliser le conflit et d'engager des recours auprès des instances internationales (Latte Abdallah, 2014b, 2014c). Suite à sa reconnaissance par l'ONU en tant qu'État non membre en septembre 2012, la Palestine a adhéré à l'ensemble des agences de l'ONU puis a ratifié le Statut de Rome en avril 2015. Depuis, l'Autorité palestinienne ainsi que différents acteurs de la société civile constituent des dossiers destinés à la Cour pénale internationale qui visent tout d'abord à faire reconnaître les crimes commis pendant la guerre de Gaza de juillet-août 2014 et à mettre juridiquement en cause la colonisation en Cisjordanie. Des démarches ont également été entreprises en vue d'obtenir l'adoption de résolutions du Conseil de Sécurité des Nations unies pour fixer un terme à l'occupation israélienne.

La société civile a quant à elle mobilisé d'autres lieux et formes de contestations. Depuis une dizaine d'années, les sites de résistance populaire et pacifique où militants palestiniens, israéliens et internationaux s'opposant de manière ritualisée et créative à la prédation territoriale se sont multipliés: Na'lin, Bil'in, Nabi Saleh, la vallée du Jourdain, Cheikh Jarrah, Qufr Qadum, Beit 'Umar, Bab al-Shams, etc. Ils ont d'ailleurs rencontré un écho médiatique et politique croissant. Parallèlement, le mouvement Boycott, Désinvestissement et Sanctions (BDS) ne cesse de monter en puissance à l'échelle internationale. Les mises en cause des entreprises internationales qui sont 
associées à des groupes israéliens implantés dans les zones commerciales, agricoles et industrielles des colonies se sont multipliées. Les adhésions à la ligne politique du BDS se sont accrues dans les cercles universitaires internationaux. Même des organisations supra-nationales comme l'Union européenne ont accentué les pressions en faveur d'une traçabilité des marchandises produites dans les colonies israéliennes. À l'échelle locale, on observe qu'à la suite de l'offensive meurtrière dans la bande de Gaza en juillet et août 2014, le boycott des produits israéliens s'est accru au sein de la société palestinienne. Des actions militantes spectaculaires ont été entreprises telles que les déversements en pleine rue du contenu de camions transportant des marchandises israéliennes, et des voix toujours plus nombreuses appellent l'Autorité palestinienne à soutenir non seulement le boycott des produits des colonies, mais aussi celui de l'ensemble des produits israéliens.

Depuis octobre 2015, ce que certains ont nommé «l'Intifada des couteaux » ou "l'Intifada de Jérusalem » a inauguré un nouveau cycle de violence. Des attaques à la voiture bélier, à l'arme blanche et plus rarement à l'arme à feu ont été perpétrées par des Palestiniens agissant le plus souvent seuls en marge des partis et des organisations collectives. Elles ont été sévèrement réprimées puisque dans la plupart des cas, les assaillants, même lorsqu'ils ont été neutralisés, ont été tués sur le coup par les forces de police ou l'armée. Certaines associations ont vivement dénoncé ces exécutions extra-judiciaires et celles d'autres Palestiniens sur les check-points et à Jérusalem dont l'implication dans des actes violents n'avait pas été prouvée. Depuis décembre 2015, la police israélienne a redéfinit ses régulations pour autoriser les officiers à ouvrir le feu sur des lanceurs de pierre, de bouteilles incendiaires ou de feux d'artifice ${ }^{3}$. Parallèlement, les arrestations se sont multipliées. Elles ont visé tout particulièrement la jeunesse palestinienne de Jérusalem et toute forme de militantisme, et notamment, les personnes actives sur les réseaux sociaux. 5000 personnes ont ainsi été arrêtées depuis octobre 2015. Une série de mesures punitives collectives ont également été prises telles que le bouclage de certaines enclaves palestiniennes, la suspension des permis de travail et de circulation, la destruction de maisons, etc. Entre octobre 2015 et la mi-juillet 2016, 214 Palestiniens, 34 Israéliens, deux Américains, un Erythréen et un Soudanais ont perdu la vie ${ }^{4}$.

Cependant, jusqu'à présent, cette «troisième Intifada »n'a pas généré de mobilisation et de réactions plus larges au sein de la société palestinienne. Elle n'a pas non plus conduit à une réorganisation mutations significatives des mécanismes de contrôle israéliens. Si les autorités israéliennes ont travaillé à circonscrire et étouffer la vague de violence en déployant pour la première fois des check-points volants au sein de la ville de Jérusalem pour filtrer les entrées et les sorties des quartiers Palestiniens ${ }^{5}$, leurs modes d'action dans le reste des Territoires occupés sont restés structurellement inchangés.

Plus récemment, des chercheurs ont appréhendé le cas israélo-palestinien à travers le cadre théorique du «colonialisme de peuplement» (settler colonialism) (Veracini, 2006, 2010 ; Collins, 2011 ; Perugini, 2009-2010). Ce colonialisme se distingue du colonialisme en général par ses objectifs. Alors que le colonialisme vise à la conquête d'un territoire, le colonialisme de peuplement cherche à s'accaparer ce territoire et à remplacer la population autochtone par une société de colons en mettant en œuvre différents types de transfert (Veracini, 2006, 2010 ; Lefevre, 2015). En outre, le colonialisme de peuplement n'est pas réductible à un événement ou une période : c'est une structure (Wolfe, 1999). Les tenants de cette approche insistent donc sur la nécessité d'établir une continuité entre la Nakba (1947-1949) qui a accompagné la création d'Israël, la conquête de la Cisjordanie et de

\footnotetext{
${ }^{3}$ Adalah, 2016, « Israeli police reveal new open-fire regulations in response to Adalah's court petition », mis en ligne le 05 juillet 2016, consulté le 19 juillet 2016, http://www.adalah.org/en/content/view/8845.

${ }^{4}$ AFP, 13 juillet 2016, «Un Palestinien tué par des garde-frontières israéliens en Cisjordanie ».

5 Peter Beaumont, «New checkpoints and fears divide Jerusalem's Jews and Palestinians », The Guardian, Wednesday 14 October 2015, consulté le 17 juillet 2016.
} 
la bande de Gaza en 1967, et les événements les plus récents, c'est-à-dire la mise en œuvre de la politique de séparation, le siège de Gaza, les confiscations de terres et les déplacements des Bédouins du sud d'Israël, etc. L'ensemble de ces événements est envisagé dans une même logique de dépossession du peuple palestinien.

Ce cadre théorique représente un premier intérêt dans la mesure où il permet de réintroduire la dimension coloniale du projet sioniste. Comme l'avait souligné Maxime Rodinson (1967), si le projet politique élaboré par Herzl a été au départ pensé comme un projet d'émancipation des Juifs d'Europe à la fin $d u X X^{\mathrm{e}}$ siècle, il s'est également construit dans un contexte politique, économique et culturel fortement dominé par l'idéologie colonialiste. C'est cette idéologie qui a conduit le sionisme politique à envisager la Palestine comme une «terre sans peuple pour un peuple sans terre » et qui continue depuis de façonner fortement les représentations que se font les Israéliens des Palestiniens (Sa'di, 1992, 1997 ; Slyomovics, 1998 ; Yacobi et Shadar, 2014).

En outre, cette approche permet aussi de rappeler le caractère fortement structurant, sur les plans territoriaux, sociaux, économiques et politiques, du processus de colonisation de peuplement qui s'est étendu après la guerre de juin 1967 à la Cisjordanie et la bande de Gaza et qui s'est accéléré depuis les années 1990. L'importance de cette colonisation de peuplement est d'ailleurs soulignée par de nombreux autres chercheurs (Efrat, 2006; Gordon, 2008; Gorenberg, 2006 ; Segal et Weizman, 2003 ; Zertal et Eldar, 2007). Le retrait de Gaza n'a d'ailleurs pas remis en cause ce processus. En réalité, ce désengagement a surtout fait diversion. N'impliquant que le retrait d'un nombre limité de colons (8 500) et laissant espérer à la communauté internationale une inversion de tendance, il a masqué le renforcement du processus de colonisation en Cisjordanie (Dawes, 2015).

Le second intérêt du paradigme de la colonisation de peuplement est de remettre en cause la validité des lectures qui présentent le conflit israélo-palestinien comme étant entré dans une phase transitoire ou postcoloniale. Apparu à partir des accords d'Oslo (1993-1999), ce type de lecture continue à influencer les récits politiques, médiatiques mais également académiques (Latte Abdallah et Parizot, 2011, 2015). En présentant le conflit comme étant entré depuis 1993 dans une phase de résolution, cette lecture transitoire favorise une analyse erronée des processus politiques, économiques et sociaux. Centrée sur les négociations, elle maintient en outre l'illusion d'une symétrie entre Palestiniens et Israéliens.

Pourtant, Palestiniens et Israéliens ne sont jamais entrés dans ce moment postcolonial. Au contraire, depuis la signature des Accords de Principe (1993) à Washington, le processus de dépossession et de confinement des Palestiniens s'est poursuivi, voire accentué (Jabary Salamanca et al., 2012, p. 4). L'attachement qu'ont manifesté les différents gouvernements israéliens à l'égard d'une solution négociée ne serait en réalité qu'un moyen de gagner du temps dans un processus de grignotage de l'espace et des ressources et de mise à l'écart de la population palestinienne (Dawes, 2015). Les négociations et les pratiques de peace-building fonctionneraient donc à présent comme un système contre-insurrectionnel visant à pacifier la population palestinienne (Turner, 2014).

Le second intérêt de l'approche en terme de colonisation de peuplement est donc, dans le contexte actuel, de remettre radicalement en cause l'horizon d'attente constitué par les négociations, tout en contestant le caractère opératoire des catégories de «guerre » et de «conflit » entre les deux parties pour décrire les processus en cours dans les territoires palestiniens occupés. Ces termes offrent en effet une vision erronée de la situation car, d'un côté, ils donnent l'image de deux entités structurellement équivalentes et évacuent l'asymétrie dans les rapports de pouvoir et, de l'autre, ils tendent à masquer les dimensions centrales de l'occupation et de la colonisation. 
Enfin les tenants du colonialisme de peuplement appellent à une recherche plus comparative. Tout comme les chercheurs qui rapprochent le régime israélien du système d'apartheid sud-africain, ou d'autres formes de régimes de séparation (Dayan, 2009), ils suggèrent de rompre avec l'idée selon laquelle le cas israélo-palestinien serait exceptionnel pour le rapprocher d'autres formes de colonialisme de peuplement en le comparant aux États-Unis, à l'Australie, au Canada, à l'Algérie française ou à l'Afrique du Sud (Jabary et al., 2013, p. 4).

\section{Les multiples acteurs du régime de mobilité}

Bien qu'il ouvre des pistes de réflexion pertinentes, notamment sur la manière de relire les trajectoires du conflit et sur la nécessité de rompre avec l'idée d'exceptionnalisme israélien, le cadre théorique du colonialisme de peuplement et la perspective d'un régime d'apartheid présentent, de notre point de vue, un certain nombre de biais. Comme toute approche trop paradigmatique et politique, celle-ci comporte le risque de masquer les spécificités des dispositifs de contrôle dans l'espace israélo-palestinien (Azoulay et Ophir, 2008).

Tout d'abord, en insistant trop sur la dimension structurelle du colonialisme, on risque d'évacuer les mutations des différentes formes de domination qui ont eu lieu au cours du temps. Si le régime d'occupation israélien présente jusqu'à aujourd'hui une forte dimension coloniale, il se distingue nettement de celui, également colonial, qui avait été mis en place de 1967 à 1987. Après l'occupation de la Cisjordanie et de la bande de Gaza, Israël s'était accaparé ces territoires et leurs ressources tout en excluant la population locale au sein de la communauté politique israélienne. Par contre, afin d'éviter toute rébellion, il avait pris en charge la gestion du système économique ainsi que des systèmes de santé et d'éducation, pour assurer un certain niveau de prospérité à la population palestinienne (Gordon, 2007, 2008). Au lendemain de la première Intifada (1987-1993), c'est un tout autre régime qui émerge. Dans le cadre des négociations, les autorités israéliennes se sont déchargées de l'administration de la population palestinienne pour la déléguer à d'autres acteurs (Autorité palestinienne, organisations et bailleurs de fonds internationaux, ONG, acteurs économiques privés). Réorganisant parallèlement leurs dispositifs de contrôle civils et militaires, elles ont pu maintenir le contrôle sur l'ensemble de la population et des territoires palestiniens tout en réduisant radicalement son coût économique et politique.

En outre, en ne comparant Israël qu'avec d'autres contextes coloniaux (Jabary et al., 2013), on risque de ne pas noter les nombreuses similitudes que présentent les dispositifs de contrôle déployés par Israël avec ceux rencontrés aujourd'hui en Europe et dans des contextes non coloniaux (Latte Abdallah et Parizot, 2011). Afin de gérer les flux de personnes et de marchandises, les dispositifs israéliens et européens reproduisent des mécanismes similaires de gestion du risque. En instaurant un accès différentiel au mouvement en fonction des populations, ils organisent des espaces inégaux de circulation, de mise à l'écart et de confinement et constituent une force majeure de stratification et de hiérarchie (Shamir, 2005, Parizot et al., 2014).

De même la construction de mur, n'est pas propre aux contextes coloniaux, mais bien à des formes de gouvernementalité post-modernes. Depuis la chute du mur de Berlin, cette technologie politique s'est largement disséminée, autant au sein des démocraties occidentales que dans des pays autoritaires tels que l'Arabie Saoudite (Vallet, 2014). La téichopolitique, c'est-à-dire la politique de construction de barrières, (Rosière et Jones, 2012), vise à mettre en scène la souveraineté de l'État à ses frontières. L'imaginaire qui est mobilisé à cet effet est celui de l'État nation moderne, conçu comme étant étendu sur un territoire homogène délimité par une frontière ligne qui lui sert d'enveloppe protectrice. 
Toutefois, comme le montre un certain nombre de recherches, la construction des murs met en œuvre une logique antinomique avec le concept de frontière-ligne. Ces dispositifs cachent en effet la fréquente projection du contrôle de l'État sur le territoire de l'Autre à travers le déploiement de systèmes complexes de surveillance. En outre, contrairement à son objectif déclaré, la construction de barrières et de murs met en œuvre des formes de délégation du contrôle qui font émerger de nouvelles formes de souveraineté. Selon Wendy Brown (2010), la construction des murs implique très souvent des interventions d'acteurs supra-nationaux et infra-nationaux, privés et publics, que ce soit dans le financement, la planification ou la mise en œuvre de ces dispositifs de contrôle. Israël ne fait pas exception : dès le début des années 2000, l'entretien du Mur et de ses points de passages entre la Cisjordanie et Israël a été confié à des organismes privés et semi-privés (Havkin, 2011, 2015). Certains checkpoints ont également bénéficié de financements étrangers (Garb, 2011, 2015). Enfin, le tracé du mur a été régulièrement révisé sous la pression des acteurs locaux et internationaux (Latte Abdallah et Parizot, 2011, 2015). En un mot, l'État d'Israël a délégué des parts de souveraineté à ses «frontières ».

La troisième limite de l'approche en termes de colonisation de peuplement est que, en invitant à se focaliser sur les rapports de pouvoir entre un État israélien colonial et les Palestiniens, ce cadre théorique détourne l'attention du rôle que peuvent jouer certains acteurs internationaux (organisations supranationales, bailleurs de fonds, ONG, sociétés privées, migrants africains, asiatiques et européens, diplomates, journalistes, chercheurs etc.). Ce faisant, cette approche risque de laisser de côté les nouvelles formes de délégation du contrôle qu'organisent le régime d'occupation israélien et les multiples acteurs qui sont, de fait, impliqués dans son fonctionnement.

Depuis le début des années 1990, Israël n'est plus seul à prendre en charge les coûts de l'occupation. Aujourd'hui, une partie conséquente des coûts financiers, administratifs et politiques sont de facto assumés par les bailleurs de fonds, des organisations internationales et par l'Autorité palestinienne. À ce niveau, l'Europe joue un rôle central. Alors qu'à l'instar d'autres organismes internationaux, elle s'était investie au début pour soutenir le processus de construction nationale palestinien (Brynen, 2000 ; Lia, 2007), l'échec des négociations et la dégradation de la situation économique et politique ont transformé son aide au développement en une prise en charge financière d'une grande part des coûts liés à l'occupation. D'autres travaux ont analysé le rôle fondamental joué par les organisations non gouvernementales qui, tout en soutenant les Palestiniens, contribuent à alléger le coût de l'occupation pour les Israéliens (Challand, 2009).

Les réformes récentes des dispositifs de séparation s'inspirent en outre de la doxa et des pratiques néo-libérales. Elles organisent une gestion managériale de l'occupation dont l'objectif est de la rendre moins couteuse économiquement et politiquement. La délégation de la gestion des checkpoints situés sur la ligne de «suture » $(\text { kav ha-tefer })^{6}$ a été confiée à des compagnies privées (Havkin, 2011, 2015, dans ce volume), tandis que la nouvelle économie pénale et carcérale, mise en place au début des années 2000, repose sur la prise en charge d'une part de ses coûts par l'Autorité palestinienne, sur la privatisation de certains services connexes à la prison et sur l'usage extensif des sanctions financières (Latte Abdallah, 2011, 2015, dans ce volume).

Des acteurs et intérêts économiques privés contribuent également à la mise en œuvre et aux réajustements de la politique coloniale de l'État en cherchant à profiter du système de zones franches, des subventions et des gains de compétitivité que permet de faire le faible niveau des coûts de production dans les colonies (Al Ghazi, 2009) ou dans les zones C (c'est par exemple le cas des supermarchés Rami Levi, qui entendent servir tout autant les colons que les populations palestiniennes). Ces acteurs bénéficient en outre d'une main d'œuvre bon marché : employés et

\footnotetext{
${ }^{6}$ Il s'agit de la zone située entre le tracé du Mur et la Ligne Verte.
} 
travailleurs palestiniens, employés des colonies appartenant à des couches défavorisées et moins payées, telles que les femmes juives orthodoxes (Al Ghazi, 2009). Outre la production agricole, les investisseurs privés ont diversifié les types de productions dans ces espaces en y implantant par exemple des sociétés high tech. Ils sont ainsi devenus des acteurs clefs du processus de colonisation. Des recherches récentes sur Jérusalem ont également montré comment la logique néolibérale de la privatisation de l'espace et de la planification urbaine peut tout à fait s'articuler avec la politique municipale de judaïsation de la ville (Braier et Yacobi, 2014).

À côté de ces acteurs formels intervient un ensemble d'acteurs informels qui sont de facto intégrés au système de régulation des mobilités humaines et marchandes. Il s'agit des réseaux de contrebande ou des passeurs qui facilitent les circulations des Palestiniens et des marchandises en contournant les dispositifs de confinement israéliens. Toutefois, motivés par des considérations économiques, ils imposent leurs propres mécanismes de régulation (Garcette, dans ce volume, Natsheh et Parizot, 2011, 2015 ; Parizot, 2013 et dans ce volume ; Pelham, 2015) modifiant ainsi le fonctionnement du régime de mobilité. Ces acteurs sont d'autant plus intégrés au fonctionnement du système de contrôle que les autorités israéliennes ont largement infiltré et instrumentalisé leurs réseaux pour développer et disséminer des réseaux d'informateurs au cœur des enclaves palestiniennes (Parizot, 2013 et dans ce volume).

\section{Étudier les mobilités pour mieux comprendre les dispositifs de séparation}

Compte tenu de la multiplicité des acteurs et de la complexité des logiques qui animent le régime d'occupation israélien, il convient d'accorder une grande attention aux interactions qu'ils entretiennent les uns avec les autres. En ce sens, notre approche s'inscrit dans le prolongement de Neve Gordon (2008) lorsqu'il proposait d'envisager les mutations des dispositifs de contrôle israéliens comme le produit des interactions, des détournements et des contradictions générées par les moyens de contrôle déployés par Israël. Ces dispositifs de contrôle englobent l'ensemble des institutions, des dispositifs légaux, des appareils bureaucratiques, des pratiques sociales et des infrastructures matérielles qui agissent à la fois sur l'individu et la population afin de produire de nouveaux comportements, de nouvelles habitudes, intérêts, goûts et aspirations. Dans une approche similaire, Adi Ophir, Michal Givoni et Sari Hanafi (2009) suggèrent d'analyser ce régime politique comme un agencement instable d'appareils et d'institutions étatiques et non étatiques, de modes de pensée et de série de technologies politiques (Ophir et al., 2009, p. 15-17).

Cependant, contrairement à ces travaux, nous nous focaliserons surtout sur les mobilités, les vécus et les interactions des acteurs à l'interface des espaces israéliens et palestiniens, à partir de la fin de la seconde Intifada (2004). L'analyse de ces circulations présente quatre avantages (Latte Abdallah et Parizot, 2011, 2015). En premier lieu, elle permet de prendre en compte les rôles que jouent l'ensemble des acteurs qui interviennent dans ces espaces : les Israéliens, les Palestiniens mais aussi les migrants africains, asiatiques, les diplomates, le personnel des ONG, les militants internationaux, etc. En second lieu, elle permet d'envisager les limites auxquelles sont quotidiennement confrontées ces populations. Cette approche offre donc une vision extrêmement précise du fonctionnement et des incohérences du régime de séparation. En troisième lieu, en se focalisant sur les pratiques ordinaires, elle donne la possibilité de cerner comment ces populations contournent ou détournent à leur avantage les contraintes imposées par les restrictions de mouvement et les mécanismes de surveillance. Car, in fine, la politique de séparation n'a pas séparé les populations vivant dans les espaces israélo-palestiniens, elle les a forcées à réajuster et transformer leurs relations. En dernier lieu, l'étude de ces interactions permet d'évaluer comment certains acteurs peuvent en retour affecter 
à leur propre échelle le fonctionnement des dispositifs de contrôle israéliens et provoquer des processus qui échappent aux prévisions de leurs promoteurs.

En bref, en partant de l'analyse de ces circulations et de ces échanges, nous tentons d'évaluer la capacité structurante et productrice du pouvoir dans ses multiples localisations et à des échelles distinctes : c'est-à-dire que nous n'envisageons pas uniquement la politique de séparation israélienne sous l'angle réducteur de ses manifestations territoriales (barrières, murs, checkpoints, etc.), mais plutôt à travers son fonctionnement. À l'instar de Didier Bigo, nous envisageons les frontières comme l'articulation de réseaux d'acteurs hétérogènes et d'opération de contrôle dont le but est de filtrer, canaliser et organiser le mouvement des personnes et des marchandises (Bigo, 2010).

Nous commencerons par envisager ces interactions au niveau des institutions. La première partie de l'ouvrage examine la mise en œuvre, l'impact et les limites de la séparation entre Israéliens et Palestiniens à travers quatre processus institutionnels. Les deux premiers chapitres abordent la réforme des dispositifs qui régulent les mouvements des Palestiniens ou organisent leur confinement: les checkpoints (Shira Havkin) et le système carcéral israélien (Stéphanie Latte Abdallah). Ils soulignent comment l'introduction de discours et de pratiques managériales dans la réforme de ces institutions fait émerger de nouvelles techniques de gouvernement des Palestiniens dont l'objectif est à la fois de réduire sensiblement les coûts politiques et économiques de l'occupation et de normaliser les dispositifs de séparation. La «civilianisation » des checkpoints, c'est-à-dire la délégation de ces points de contrôle à des compagnies privées, est d'ailleurs envisagée par les autorités israéliennes comme devant être un projet consensuel, c'est-à-dire un moyen de dépolitiser le contrôle et de pacifier les relations avec une population palestinienne maintenue pourtant sous surveillance. La réforme du système carcéral vise quant à elle à décharger une partie des coûts de détention vers l'Autorité palestinienne, des organismes privés locaux et les bailleurs de fonds internationaux. Mais les évolutions de la justice militaire et du système carcéral génèrent des effets qui affectent le fonctionnement pratique de ces institutions. Stéphanie Latte Abdallah décrit ainsi comment les interactions des juges et procureurs militaires, des fonctionnaires, israéliens et palestiniens, des avocats, des prévenus et de leurs familles font émerger un véritable «business du carcéral ».

Dans le prolongement de ces deux premiers articles, Philippe Bourmaud offre un éclairage sur l'impact de ces dispositifs sur la constitution du tissu institutionnel palestinien. L'auteur analyse la difficile organisation d'un système cohérent de formation et de soins médicaux dans des territoires palestiniens discontinus. Toutefois, s'il souligne bel et bien le rôle que jouent les entraves à la mobilité des personnes dans la multiplication des facultés de médecine et la segmentation de leurs réseaux, il insiste également sur la nécessité de prendre en compte l'influence du contexte institutionnel palestinien et, notamment, celle des rapports de force entre les universités et l'Autorité palestinienne. Enfin, Irène Salenson revient, dans un quatrième chapitre, sur la mise en place de la gestion coordonnée de l'eau entre Israéliens et Palestiniens. Créé au milieu des années 1990, au cours de la période « intérimaire », le dispositif de gestion des ressources hydriques a perduré depuis cette date faute d'accord permanent. Cette situation a non seulement permis aux Israéliens de conserver un contrôle partiel sur l'eau, mais elle a également favorisé l'intervention croissante des agences internationales soit pour suppléer les organisations palestiniennes embryonnaires, soit pour jouer le rôle de médiatrices. En somme, ces premiers chapitres montrent que si les politiques de séparation et de confinement des Palestiniens sont pensées et organisées par les autorités israéliennes, leur mise en œuvre et les effets qu'elles ont sur le terrain sont constamment réajustés par l'intervention de différents types d'acteurs qui poursuivent leurs propres intérêts.

Ces acteurs formels et informels jouent parfois un rôle déterminant dans les reconfigurations des relations entre Israéliens et Palestiniens. C'est ce qu'envisage la deuxième partie de cet ouvrage. À 
travers l'étude des recompositions du secteur oléicole et des filières de passeurs qui assurent la traversée clandestine des Palestiniens en Israël, Arnaud Garcette et Cédric Parizot mettent en lumière les adaptations, les contournements et les réappropriations du régime de séparation par des Palestiniens et certains Israéliens. Il ne s'agit pas simplement ici d'insister sur la capacité de résilience des Palestiniens, mais plutôt de souligner qu'ils sont parfois au cœur des mécanismes de régulation. En décrivant la réorganisation des réseaux d'intermédiaires dans la commercialisation et l'exportation des produits oléicoles ou dans l'organisation du passage clandestin vers Israël, ces deux auteurs montrent comment des civils israéliens, mais aussi Palestiniens s'imposent de facto comme des autorités informelles contribuant à la régulation des mouvements des marchandises et des personnes. Enfin, en mettant en lumière des réseaux de médiation associant des Israéliens, des Palestiniens et des étrangers, ils montrent que la politique de séparation génère parfois des groupes d'intérêts qui n'épousent pas les frontières culturelles, statutaires et politiques délimitées par les catégories institutionnelles.

La troisième partie approfondit cette dernière question en déplaçant le regard vers des expériences plus individuelles. L'enjeu est tout d'abord de comprendre comment les populations palestiniennes construisent et mettent en mot leurs pratiques et vécus personnels, voire intimes, des frontières. Véronique Bontemps décrit la manière dont les ouvriers palestiniens qui travaillent en Israël sans permis parlent de leur traversée et de leur expérience collective de précarité et d'humiliation qui les sépare des autres Palestiniens. Sarah Memmi analyse les adaptations et les contournements que déploient, pour continuer à vivre ensemble, les couples Palestiniens séparés par les frontières administratives entre la Cisjordanie, la bande de Gaza, Jérusalem et Israël. Ces deux chapitres montrent non seulement la multiplicité des frontières (territoriales, statutaires, identitaires) qui structurent la vie des Palestiniens, mais également les nouvelles hiérarchies qu'elles organisent entre eux.

La quatrième partie traite des expériences militantes de part et d'autre de la Ligne verte. Elle illustre la complexité des interactions entre les Palestiniens d'Israël et les Palestiniens des Territoires occupés (Elizabeth Marteu), ainsi qu'entre ces derniers et les Israéliens juifs animant les luttes LGBTQ (Valérie Pouzol) ou ceux engagés contre l'occupation (Karine Lamarche). Les deux premiers chapitres soulignent le poids déterminant du politique dans les reconfigurations à l'œuvre. Les transformations du militantisme LGBTQ montrent comment les groupes militants palestiniens (présents à Jérusalem-Est et en Israël) ont réinvesti le champ politique palestinien à partir des années 2000, en mettant sur un même plan l'affirmation de sexualités ou d'identités sexuelles différentes et un positionnement politique radical qui rompt entièrement avec le champ militant israélien. La cause palestinienne est également devenue un espace commun d'identification politique dans les dynamiques décrites par Elizabeth Marteu à propos des associations palestiniennes des deux côtés de la ligne verte. Toutefois, dans ce dernier cas, les pratiques et les répertoires d'action sont distincts et se développent de façon autonome. Enfin, Karine Lamarche étudie les vécus de «passeurs de frontières » israéliens à savoir les militants contre l'occupation qui se rendent en Cisjordanie. Elle analyse comment leurs expériences transforment leurs représentations du monde, redessinent profondément les lignes de fractures et modifient leur positionnement au sein de la société israélienne.

Pour achever ce processus de déconstruction du fonctionnement de la politique et des récits sur la séparation, les deux dernières parties de ce livre proposent un détour vers les expériences d'autres populations que les Israéliens et les Palestiniens des territoires occupés par Israël en 1967. Ce décentrage permet tout d'abord de mettre en évidence que, bien qu'étant hégémonique le discours articulé autour de la séparation entre Israéliens et Palestiniens coexiste avec d'autres constructions et d'autres pratiques des espaces et de la frontière. Il s'agit aussi de rappeler que les espaces israélo- 
palestiniens ne sont pas coupés du Moyen-Orient et du reste du monde, mais qu'ils y sont intégrés par un ensemble de processus dont les mobilités régionales et internationales des populations qui les traversent.

C'est ainsi que dans la cinquième partie Adoram Schneidleder et Daniel Meier envisagent la frontière israélo-libanaise à partir des vécus et des discours de deux groupes de Libanais vivant en Galilée (des femmes ayant épousé des Israéliens - juifs ou arabes - et d'anciens miliciens de l'Armée du Liban Sud) pour le premier et des réfugiés palestiniens du Liban pour le second. Leurs contributions permettent de mettre en perspective d'autres constructions de la nation et des frontières. Ancrées dans la mémoire des lieux, dans les histoires collectives et les vécus individuels et émotionnels, celles-ci donnent corps à des espaces perçus et parcourus qui s'étendent par-delà la frontière internationale.

Dans la sixième partie, Lisa Anteby décentre l'analyse plus au sud pour observer les acteurs qui construisent, traversent et négocient la frontière israélo-égyptienne : les passeurs bédouins dans le Sinaï et dans le Néguev, les soldats égyptiens et israéliens, les législateurs, mais aussi les ONG, les municipalités israéliennes, ainsi que les réseaux de migrants soudanais ou érythréens. L'auteure met ainsi en perspective la complexité des mécanismes qui régulent formellement et informellement l'arrivée des migrants et leur accueil en Israël. Elle souligne également les formes concurrentes de représentation de la frontière qui prévalent dans cette région. Enfin, pour clore cette partie, William Berthomière et Caroline Rozenholc nous conduisent à Tel Aviv, dans les rues des quartiers de Florentin et Neve Sha'anan, qui rassemblent de nombreux migrants africains, asiatiques et européens. Ils montrent comment les dynamiques urbaines et migratoires recomposent des frontières sociales et les espaces de cohabitation au cœur de la «bulle » israélienne.

Jérusalem, Aix-en-Provence, juillet 2016

\section{RÉFÉRENCES BIBLIOGRAPHIQUES}

ABU ZAHRA, Nadia, 2007, Legal Geographies in Palestine: Identity Documentation, Dispossession, Repression and Resistance, thèse de doctorat, Université d'Oxford, sous la direction de Tony Lemon et Dawn Chatty.

AL GHAZI, Gadi, 2009, « Matrix in Bil'in: Colonial Capitalism in the Occupied Territories », in Adi Ophir, Michal Givoni et Sari Hanafi (dir.), The Power of Inclusive Exclusion: Anatomy of Israeli Rule in the Occupied Palestinian Territories, Cambridge, Zone Books, p. 519-434.

AMIDROR (M. G. reserve), Yaakov, 2007, Counterinsurgency War: The Israeli Experience, Jérusalem, Jerusalem Center for Public Affairs.

ARIELI, Shaul et Michael SFARD, 2008, The Wall of Folly, Tel Aviv, Yediot Aharonot. (en hébreu).

AZOULAY, Ariella et Adi OPHIR, 2008, This Regime Which is Not One: Occupation and Democracy between the Sea and The River (1967 - ), Tel-Aviv, Resling. (en hébreu).

BIGO, Didier, 2010, «Frontières, territoire, sécurité, souveraineté », Ceriscope, 30 novembre 2010, http://spire.sciencespo.fr/hdl:/2441/eu4vqp9ompqllr09i45ao4c3h/resources/frontieresterritoire-securite-souverainete.pdf, consulté le 12 septembre 2014. 
BRAIER, Michal et Haim YACOBI, 2014, «On Colonialism, Neoliberalism and Nationalism: Dwelling in Jerusalem », Présentation orale, A divided city? Rethinking Borders, settlements and inter-communal interactions in metropolitan Jerusalem, Tourtour, Fondation des Treilles, 4-7 novembre 2014.

BOLE-RICHARD, Michel, 2013, Israël: le nouvel apartheid, Mayenne, Les liens qui libèrent.

BRYNEN, Rex, 2000, A Very Political Economy: Peacebuilding and Foreign Aid in the West Bank and Gaza, Washington DC, United States Institute of Peace.

CHALLAND, Benoit, 2009, Palestinian Civil Society, Foreign Donors and the Power to Promote or Exclude, Oxon/New York, Routledge.

COHEN, Samy, 2009, Tsahal à l'épreuve du terrorisme, Paris, Le Seuil.

COLLINS, John, 2008, « Dromocratic Palestine », Middle East Report, 248, hiver, p. 8-13.

COLLINS, John, 2011, Global Palestine, Londres, Hurst\&Co.

CYPEL, Sylvain, 2005, Les emmurés. La société israélienne dans l'impasse, Paris, La Découverte.

DAWES, Simon, 2015, «The Digital Occupation of Gaza: An Interview with Helga TawilSouri », Networking Knowledge 8(2), URL: http://ojs.meccsa.org.uk/index.php/netknow/article/view/374/204, consulté le 13 juillet 2015.

DAYAN, Hilla, 2009, «Regimes of Separation: Israel/Palestine and the Shadow of Apartheid », in Adi Ophir, Michal Givoni et Sari Hanafi (dir.), The Power of Inclusive Exclusion: Anatomy of Israeli Rule in the Occupied Palestinian Territories, Cambridge, Zone Books, p. 281-322.

DUGARD, John, 2007, Report of the Special Rapporteur on the situation of human rights in the Palestinian territories occupied since 1967, Human Rights Council | Fourth Session, January 29.

EFRAT, Elisha, 2006, The West Bank and Gaza Strip: A Geography of Occupation and Disengagement, Londres, New York, Routledge.

ENDERLIN, Charles, 2002, Le rêve brisé. Histoire de l'échec du processus de paix au ProcheOrient : 1995-2002, Paris, Fayard.

FARSAKH, Leila, 2002, «Palestinian Labor Flows to the Israeli Economy: A Finished Story? », Journal of Palestine Studies, 32(1), p. 14-15.

FMEP, 2011, « Settler Population Growth East and West of the Separation Barrier, 2000-2010 », http://fmep.org/resource/settler-population-growth-east-and-west-of-the-separation-barrier-2000$\underline{2010 /}$, consulté le $1^{\text {er }}$ juin 2015.

FMEP, 2011, «Settler Population East of the Separation Barrier, 1994-2010», URL: http://fmep.org/resource/settler-population-east-of-the-separation-barrier-1994-2010/, consulté le $1^{\text {er }}$ juin 2015.

FMEP, 2013, «West Bank Settlements Population - 1999-2012, URL: http://fmep.org/resource/west-bank-settlements/, consulté le $1^{\text {er }}$ juin 2015. 
GORDON, Neve, 2007, « Of Dowries and Brides: A Structural Analysis of Israel's Occupation », New Political Science, 29(4), p. 453-478.

GORDON, Neve, 2008, Israel's Occupation, Berkeley, University of California Press.

GRINBERG, Lev, 2011, «Discours économique et construction des frontières dans l'espace israélo-palestinien depuis 1967 », in Latte Abdallah, S., Parizot, C. (dir.), À l'ombre du Mur. Israéliens et Palestiniens entre occupation et séparation, Arles, MMSH/Actes Sud, p. 105-123.

HANDEL, Ariel, 2009, "Where, Where to and When in the Occupied Territories? An Introduction to Geography of Disaster », in M. Givoni, S. Hanafi, A. Ophir (dir.), The Power of Exclusive Inclusion, New York, Zone Books, p. 179-222.

HANIEH, Adam, 2006, «The Politics of Curfew in the Occupied Territories », in Joel Beinin et Rebecca L. Stein (dir.), The Struggle for Sovereignty: Palestine and Israel 1993-2004, Stanford California, Stanford University Press, p. 324-337.

HASS, Amira, 2001, Boire la mer à Gaza, Paris, La Fabrique.

HASS, Amira, 2002, «Israel's Closure Policy: An Ineffective Strategy of Containment and Repression », Journal of Palestine Studies, 31(3), p. 5-20.

HAVKIN, Shira, 2011, «La privatisation des checkpoints : quand l'occupation militaire rencontre le néolibéralisme ", in Latte Abdallah, S., Parizot, C. (dir.), À l'ombre du Mur. Israéliens et Palestiniens entre occupation et séparation, Arles, MMSH/Actes Sud, p. 51-72.

HAVKIN, Shira, 2015, «Outsourcing the Checkpoints: When Military Occupation Encounters Neoliberalism », in Latte Abdallah, S., Parizot, C. (dir.), Israelis and Palestinians in the Shadows of the Wall. Spaces of Separation and Occupation, Farnham, Ashgate, p. 25-38.

JABARY SALAMANCA, Omar, QATO, Mezna, RABIE, Kareem et Sobhi SAMOUR, 2012, «Introduction », Past is Present: Settler Colonialism in Palestine, Settler Colonial Studies, 2, 1, p. 18.

KEMP, Adriana et Rebecca RAIJMAN, 2008, Migrants and Workers: The Political Economy of Labor Migration in Israel, Jérusalem, Hakibutz Hameuchad-Van Leer Institute (en hébreu).

LATTE ABDALLAH, Stéphanie, 2011, « Déni de frontières. Toile carcérale et management des prisonniers politiques palestiniens après Oslo (1993-2010) », in Latte Abdallah, Stéphanie, Parizot, Cédric (dir.), À l'ombre du Mur. Israéliens et Palestiniens entre occupation et séparation, Arles, MMSH/Actes Sud, p. 73-101.

LATTE ABDALLAH, Stéphanie, 2012, «Les Palestiniens dans la toile carcérale », Le Monde Diplomatique juin 20 [traduit et publié dans les éditions anglaise et arabe].

LATTE ABDALLAH, Stéphanie, 2014a, «Entre dedans et dehors : vécus parentaux des détenus politiques palestiniens en Israël », Champ pénal/Penal Field, Numéro spécial, XI Parentalités enfermées/Detained Parenthood, Coline Cardi et Stéphanie Latte Abdallah (dir.), URL: www.champpenal.revues.org.

LATTE ABDALLAH, Stéphanie, 2014b, «Washington s'engage mais Israéliens et Palestiniens sont pessimistes, entretien avec Stéphanie Latte Abdallah (propos recueillis par Sybille de 
Larocque)», JOL presse, February 10, URL: http://www.jolpress.com/israel-palestinejerusalemcolonies-colonisation-paix-mahmoud-abbasnetanyahu-article-824342.html, consulté le 30 mai 2014

LATTE ABDALLAH, Stéphanie, 2014c, « Au fil des années, Israël est de plus en plus isolé sur la scène internationale, entretien avec Stéphanie Latte Abdallah (propos recueillis par Sybille de Larocque) », JOL presse, February 12, URL : http://www.jolpress.com/israel-palestine-coloniesjerusalemrefugies-frontieres-paix-john-kerryarticle-824343.html, consulté le 30 mai 2014

LATTE ABDALLAH, Stéphanie, 2015, «Denial of Borders: The Prison Web and the Management of Palestinian Political Prisoners after the Oslo Accords (1993-2013)», in Latte Abdallah, S., Parizot, C. (dir.), Israelis and Palestinians in the Shadows of the Wall. Spaces of Separation and Occupation, Farnham, Ashgate, p. 39-56.

LATTE ABDALLAH, Stéphanie, PARIZOT, Cédric (dir.), 2011, À l'ombre du Mur. Israéliens et Palestiniens entre séparation et occupation, Arles, MMSH/Actes Sud.

LATTE ABDALLAH, Stéphanie, PARIZOT, Cédric (dir.), 2015, Israelis and Palestinians in the Shadows of the Wall. Spaces of Separation and Occupation, Farnham, Ashgate.

LEBRUN, Céline et Julien, SALINGUE, 2013, Israël, un État d'apartheid ?, Paris, L'Harmattan.

LEFEVRE, Tate A., 2015, «Settler Colonialism », Anthropology, Oxford Bibliography, DOI: 10.1093/OBO/9780199766567-0125, consulté le 13 juillet 2015.

LEGRAIN, Jean-François, 1996, «La Palestine: de la terre perdue à la reconquête du territoire », Cultures \& Conflits, 21-22, p. 171-221.

LEGRAIN, Jean-François, 1997, «Palestine: Les bantoustans d'Allah », in Bocco, R., Destremau, B. et J. Hannoyer (dir.), Palestine, Palestiniens : territoire national, espaces communautaires, Beyrouth, CERMOC, p. 85-101.

LENTIN, Ronit, 2008, Thinking Palestine, Londres, Zed Books.

LIA, Brynjar, 2006, A Police without a State. A History of the Palestinian Security Forces in the West Bank and Gaza, Ithaca, Reading.

NATSHEH, Basel et PARIZOT, Cédric, 2011, « Du kit-kat au 4x4 : la séparation sous l'angle du trafic de marchandises entre Israël et la Cisjordanie », in Latte Abdallah, Stéphanie, Parizot, Cédric (dir.), À l'ombre du Mur. Israéliens et Palestiniens entre occupation et séparation, Arles, Actes Sud, p. 153-184.

PARIZOT, Cédric, 2009, «Temporalités et perceptions de la séparation entre Israéliens et Palestiniens », Bulletin $d u$ CRFJ, 20, mis en ligne le 10 mars 2010, URL: http://bcrfj.revues.org/6291?lang=en, consulté le 05/05/2014.

PARIZOT, Cédric, 2010, «Séparation et régime de mobilité entre Israël et la Cisjordanie (20002009): Réglementations, pratiques et acteurs », in Esther Benbassa (dir.), Israël-Palestine: les enjeux d'un conflit, Paris, CNRS, p. 129-146. 
PARIZOT, 2013, «A Facilitator can hide another: The Informal Dimensions of the Israeli Permit System of Access and Movementin the Occupied Palestinian Territories (1990-2010)», <halshs01116263>, URL: https://halshs.archives-ouvertes.fr/halshs-01116263/document

PARIZOT, Cédric, AMILHAT-SZARY, Anne Laure, POPESCU, Gabriel, ARVERS, Isabelle, CANTENS, Thomas, CRISTOFOL, Jean, MAI, Nicola, MOLL, Joana, VION, Antoine, 2014, « The antiAtlas of Borders, A Manifesto », Journal of Borderlands Studies, 29, 4, p. 503-512. http://www.tandfonline.com/doi/abs/10.1080/08865655.2014.983302\#.VQETivmG-MH

OLMSTED, Jennifer, 2009, «Locked in, Locked out of Work », Apartheid and Beyond, Middle East Report, 253, p. 28-31.

OPHIR, Adi, GIVONI, Michal, HANAFI, Sari (dir.), 2009, The Power of Inclusive Exclusion. Anatomy of Israeli Rule in the Occupied Palestinian Territories, New York, Zone Books.

PEACE NOW, 2013, Settlements and the Netanyahu Government:A Deliberate Policy of Undermining the Two State Solution, mis en ligne le 16 janvier, http://peacenow.org.il/eng/sites/default/files/summary-of-4-years-of-netanyahu-government.pdf, consulté le 9 juillet 2015.

PAPPE, Ilan (dir.), 2015, Israel and South Africa. The Many Faces of Apartheid, Chicago, Zed Books Ltd.

PELHAM, Nicolas, 2015, « The Rise and Fall of Gaza's Tunnel Economy (2007-2014) », in Latte Abdallah, Stéphanie, Parizot, Cédric (dir.), Israelis and Palestinians in the Shadows of the Wall. Spaces of Separation and Occupation, Farnham, Ashgate, p. 129-144.

PERUGINI, Nicola, 2009-2010, "The Frontier is Where the Jews Live: A Case of Israeli "Democratic Colonialism" ", Journal of Law and Social Research (JLSR), 1, 1, p. 73-90.

PETEET, Julie 2008, «Stealing Time », Middle East Report: Waiting, the Politics of Time in Palestine, 248 Autumn, p. 14-17.

PETTI, Alessandro, 2008, «Asymmetries », in Farquharson, Alex (dir.), The Impossible Prison, Nottingham, Centre for Contemporary Art.

RAZOUX, Pierre, 2006, Tsahal: nouvelle histoire de l'armée israélienne, Paris, Perrin.

ROSIERE, Stéphane et JONES, Reece, 2012 «Teichopolitics: Re-considering Globalisation Through the Role of Walls and Fences", Geopolitics, 17:1, p. 217-234

RODINSON, Maxime, 1967, «Israël, fait colonial ?», in Sartre, Jean-Paul (dir.), Les temps modernes, Le conflit israélo-arabe, 253 bis, p. 17-88.

ROY, Sara, 1999, « De-development Revisited: Palestinian Economy and Society Since Oslo », Journal of Palestine Studies, 28 (3), p. 64-82.

SA'DI, Ahmad, 1992, «Between State Ideology and Minority National Identity: Palestinians in Israel and in Israeli Social Science Research », Review of Middle Eastern Studies, 5, p. 110-130.

SA'DI, Ahmad, 1997, «Modernization as an Explanatory Discourse of Zionist-Palestinian Relations », British Journal of Middle Eastern Studies, 24 (1), p 25-48. 
SEGAL, Rafi et WEIZMAN, Eyal (dir.), 2003, Civilian Occupation: The Politics of Israeli Architecture, Tel Aviv, Babel et Londres, New York, Verso.

SHAMIR, Ronen, 2005, «Without Borders? Notes on Globalization as a Mobility Regime », Sociological Theory, 23(2), p. 197-217.

SLYOMOVICS, Susan, 1998, The Object of Memory: Arab and Jew Narrate the Palestinian Village, Philadelphie, University of Pennsylvania Press.

SMITH, Charles D., 2007, Palestine and the Arab-Israeli Conflict; San Martin's, Bedford.

TOENSING, Chris, 2009, «From the Editor», Middle East Report, Apartheid and Beyond, 253, $\mathrm{p} 1$.

TROTTIER, Julie, 2007, «A wall, water and power: the Israeli "separation fence" », Review of International Studies, 33, p. 105-127.

UNOCHA, 2012, The Humanitarian Impact Of Israeli Settlement Policies, December, URL: http://unispal.un.org/pdfs/OCHA_HumImpact-Settlements.pdf, accessed 15/04/2014.

VALLET, Elisabeth, 2014, Borders, Fences and Walls, Farnham, Ashgate.

VERACINI, Lorenzo, 2006, Israel and Settler Society, London/Ann Arbor, Pluto Press.

VERACINI, Lorenzo, 2010, Settler Colonialism. A Theoretical Overview, Londres, Palgrave Macmillan.

WOLFE, Patrick, 1999, Settler Colonialism and the Transformation of Anthropology. The Politics and Poetics of an Ethnographic Event, Londres and New York, Cassel.

YACOBI, Haim et SHADAR, Hadas, 2014, «The Arab Village: a genealogy of (post)colonial imagination », The Journal of Architecture, 19, 6, p. 975-997.

ZERTAL, Edith et ELDAR, Akiva, 2007, Lords of the Land: The War over Israel's Settlements in the Occupied Territories, 1967-2007, New York, Nation Boo.

\title{
Table des Matières
}

\section{ISRAËL-PALESTINE : L'ILLUSION DE LA SÉPARATION}

\author{
Table des matières
}

Stéphanie Latte Abdallah et Cédric Parizot 
«Introduction : De la séparation aux mobilités. Changer de regard sur l'occupation israélienne en Palestine »

\section{I - OCCUPATION ET ARCHITECTURES DU MOVEMENT}

\section{Stéphanie Latte Abdallah}

«L'incarcération politique des Palestiniens comme paradigme du régime de mobilité » Shira Havkin

«La « civilisation » des checkpoints. Consensus néolibéral et poursuite de la guerre par d'autres moyens »

Irène Salenson

«Eau sans frontières? Acteurs israéliens, palestiniens et internationaux du secteur de l'eau en Cisjordanie (1995-2014)»

\section{Philippe Bourmaud}

«Une architecture institutionnelle à l'épreuve de la segmentation territoriale. La formation médicale dans les Territoires palestiniens occupés depuis 1967 »

\section{II - ÉCONOMIE DES MOBILITÉS INFORMELLES}

\section{Cédric Parizot}

«Une économie du contrôle «non documentée ». Travailleurs, passeurs et autorités dans le sud israélo-palestinien (2004-2010)»

\section{Arnaud Garcette}

«L'oléiculture palestinienne au pied du mur: les acteurs sociaux-économiques face aux dispositifs de séparation israéliens en Cisjordanie (2000-2012)»

\section{III - ÊTRE(S) FRONTALIERS}

\section{Véronique Bontemps}

«Tu dois prendre le risque, tu n'as pas le choix ». Entre précarisation et décision individuelle, récits d'ouvriers palestiniens travaillant en Israël »

\section{Sarah Memmi}

«Les couples palestiniens à l'épreuve de la politique de séparation »

\section{IV- ENTRE-DEUX MILITANTS}

\section{Elizabeth Marteu}

«Les associations palestiniennes d'Israël. Entre solidarité et différenciation à l'égard des associations palestiniennes des Territoires occupés »

\section{Karine Lamarche}

« Israël-Palestine, et retour. À la rencontre des militants israéliens contre l'occupation »

\section{Valérie Pouzol}

«De l'Eden gay au front pionnier. Constructions de la «frontière » dans les luttes et discours militants LGBTQ en Israël et en Palestine (1988-2012)»

\section{V- L'AUTRE FRONTIÈRE}

\section{Adoram Schneidleder}

«Les Libanais de Galilée. Pratiques et production d'une frontière israélo-libanaise »

\section{Daniel Meier}


«La frontière israélo-libanaise vécue par les réfugiés palestiniens du Liban : entre stratégies de contournement et Palestine rêvée »

\section{VI- ISRAEL DANS L'ESPACE MIGRATOIRE INTERNATIONAL}

\section{Lisa Anteby}

« Migrations d'asile et nouveaux acteurs de la frontière israélo-égyptienne » Berthomière William \& Rozenholc Caroline

«Pour une lecture des processus de recomposition des frontières sociales depuis Tel Aviv » 